\title{
Targeted Molecular Iron Oxide Contrast Agents for Imaging Atherosclerotic Plaque
}

\author{
Rhiannon J. Evans, 1,2, Begoña Lavin², Alkystis Phinikaridou², Kok Yean Chooi³, Zahra Mohri ${ }^{3}$ Eunice Wong',4, Joseph J. \\ Boyle ${ }^{4}$, Rob Krams³ ${ }^{3}$, René Botnar², Nicholas J. Long ${ }^{1 凶}$ \\ 1. Department of Chemistry, MSRH Building, Imperial College London, White City Campus, 80 Wood Lane, White City, London, W12 0BZ, UK \\ 2. School of Biomedical Engineering and Imaging Science, St. Thomas's Hospital, King's College London, London, SE1 7EH, UK. \\ 3. Department of Bioengineering, Imperial College London, South Kensington, London, SW7 2AZ, UK. \\ 4. National Heart and Lung Institute, ICTEM Building, Imperial College London, Hammersmith Campus, Du Cane Rd, London, W12 0NN, UK. \\ $\triangle$ Corresponding author: Nicholas J. Long, Department of Chemistry, MSRH Building, Imperial College London, White City Campus, 80 Wood Lane, White City, London, W12 0BZ, UK.
}

(C) The author(s). This is an open access article distributed under the terms of the Creative Commons Attribution License (https://creativecommons.org/licenses/by/4.0/). See http://ivyspring.com/terms for full terms and conditions.

Received: 2020.03.05; Accepted: 2020.05.06; Published: 2020.05.30

\begin{abstract}
Overview: Cardiovascular disease remains a leading cause of death worldwide, with vulnerable plaque rupture the underlying cause of many heart attacks and strokes. Much research is focused on identifying an imaging biomarker to differentiate stable and vulnerable plaque. Magnetic Resonance Imaging (MRI) is a non-ionising and non-invasive imaging modality with excellent soft tissue contrast. However, MRI has relatively low sensitivity (micromolar) for contrast agent detection compared to nuclear imaging techniques. There is also an increasing emphasis on developing MRI probes that are not based on gadolinium chelates because of increasing concerns over associated systemic toxicity and deposits'. To address the sensitivity and safety concerns of gadolinium this project focused on the development of a high relaxivity probe based on superparamagnetic iron oxide nanoparticles for the imaging of atherosclerotic plaque with MRI. With development, this may facilitate differentiating stable and vulnerable plaque in vivo.
\end{abstract}

Aim: To develop a range of MRI contrast agents based on superparamagnetic iron oxide nanoparticles (SPIONs), and test them in a murine model of advanced atherosclerosis.

Methods: Nanoparticles of four core sizes were synthesised by thermal decomposition and coated with poly(maleicanhydride-alt-1-octadecene) (PMAO), poly(ethyleneimine) (PEI) or alendronate, then characterised for core size, hydrodynamic size, surface potential and relaxivity. On the basis of these results, one candidate was selected for further studies. In vivo studies using $10 \mathrm{~nm}$ PMAO-coated SPIONs were performed in ApoE-/- mice fed a western diet and instrumented with a perivascular cuff on the left carotid artery. Control ApoE-- mice were fed a normal chow diet and were not instrumented. Mice were scanned on a 3T MR scanner (Philips Achieva) with the novel SPION contrast agent, and an elastin-targeted gadolinium agent that was shown previously to enable visualisation of plaque burden. Histological analysis was undertaken to confirm imaging findings through staining for macrophages, CX3CL1, elastin, tropoelastin, and iron.

Results: The lead SPION agent consisted of a $10 \mathrm{~nm}$ iron oxide core with poly (maleicanhydride-alt-1-octadecene), $\left(-36.21 \mathrm{mV}, \mathrm{r}^{2} 18.806 \mathrm{mmol}^{-1 / \mathrm{s}^{-1}}\right)$. The irregular faceting of the iron oxide core resulted in high relaxivity and the PMAO provided a foundation for further functionalisation on surface $-\mathrm{COOH}$ groups. The properties of the contrast agent, including the negative surface charge and hydrodynamic size, were designed to maximise circulation time and evade rapid clearance through the renal system or phagocytosis. In vitro testing showed that the SPION agent was non-toxic.

In vivo results show that the novel contrast agent accumulates in similar vascular regions to a gadolinium-based contrast agent (Gd-ESMA) targeted to elastin, which accumulates in plaque. There was a significant difference in SPION signal between the instrumented and the contralateral non-instrumented vessels in diseased mice $(p=0.0411$, student's t-test), and between the instrumented diseased vessel and control vessels $(p=0.0043,0.0022$, student's $t$-test). There was no significant difference between the uptake of either contrast agent between stable and vulnerable plaques ( $p=0.3225$, student's t-test). Histological verification was used to identify plaques, and Berlin Blue staining confirmed the presence of nanoparticle deposits within vulnerable plaques and co-localisation with macrophages.

Conclusion: This work presents a new MRI contrast agent for atherosclerosis which uses an under-explored surface ligand, demonstrating promising properties for in vivo behaviour, is still in circulation 24 hours post-injection with limited liver uptake, and shows good accumulation in a murine plaque model.

Key words: MRI contrast agents, vulnerable plaque, atherosclerosis, superparamagnetic iron oxide nanoparticles 


\section{Introduction}

Cardiovascular disease (CVD), including myocardial infarction and stroke, remains a leading cause of death and long-term disability worldwide [2], costing the UK in excess of $€ 26$ billion per year to treat [3]. The high mortality is mainly caused by coronary atherosclerosis and the high morbidity by carotid atherosclerosis and associated stroke. Atherosclerosis is a chronic, inflammatory, lipiddriven disease of the artery walls. Atherosclerotic plaques are classified in a variety of ways and here we adopt the simple difference between vulnerable and stable plaques, which is based on their propensity to rupture with ensuing thrombosis that might occlude the lumen.

A major challenge for medical imaging is the development of a contrast agent which can differentiate between vulnerable and stable plaques, to identify rupture-prone lesions and perhaps to enable treatment to trigger plaque stabilisation or regression and prevent subsequent cardiovascular events. This is particularly important given the asymptomatic nature of vulnerable plaque, and the low survival rates for out-of-hospital cardiac arrests (as measured by patients subsequently discharged alive from hospital) remaining below 10\% [3].

The current gold-standard for human atherosclerosis imaging is Optical Coherence Tomography (OCT) which can differentiate between plaque phenotypes, but which is an invasive technique. Computer tomography has also shown great promise in vulnerable plaque detection but exposes patients to inonizing radiation and thus is not ideal for repeat exams [4].

Magnetic Resonance Imaging (MRI) is a non-invasive imaging technique with excellent resolution which does not use ionising radiation to generate excellent soft tissue contrast. To further enhance contrast between normal and diseased tissues, or to obtain molecular and cellular information, MR contrast agents can be used. By far the majority of MRI contrast agents are based on gadolinium-chelates which generate positive $T_{1}$ contrast through paramagnetism. However, increasingly, alternatives to gadolinium are being sought due to toxicity concerns particularly in patients with kidney disease, and recent studies which have found gadolinium deposits in patients' brains $[1,5,6]$.

Commercial, off-the-shelf, non-targeted SPIONs have been previously used to image atherosclerotic plaques in patients $[7,8]$. Whilst these studies indicate that SPIONs successfully image atherosclerotic plaques, the exact correlation with plaque phenotype is less clear.

The aim of this study was to develop a range of MRI contrast agents based on superparamagnetic iron oxide nanoparticles (SPIONs) with different surface functionalisation, and test them in a murine model of advanced atherosclerosis to determine whether a long-circulating, non-toxic SPION contrast agent might offer an alternative to invasive intravascular OCT or ultrasound, and to more traditional gadolinium-containing MRI contrast agents for the detection of vulnerable plaque.

\section{Results and Discussion}

\section{Contrast agent development}

SPIONs were synthesised using the thermal decomposition method first reported by Sun et. al. [9]. Four different size cores $(6,8,10,12 \mathrm{~nm})$ were grown to examine the relationship between core size and relaxivity. The hydrophobic particles were phase transferred using three different ligand systems, poly (maleic anhydride-alt-1-octadecene) (PMAO) [10], alendronate [11], and poly(ethyleneimine) (PEI). The surface ligands all offered different characteristics, comparing encapsulation (PMAO), and ligand exchange (alendronate and PEI), different surface groups (-COOH and $\left.-\mathrm{NH}_{2}\right)$, as well as newer (PMAO) and more established ligands (PEI).

The nanoparticles were characterised in terms of core size, hydrodynamic size, surface charge, and relaxivity. Hydrodynamic size and surface charge are both important considerations in predicting and governing in vivo behaviour. Long circulation times allow the contrast agent to accumulate in plaque through a combination of phagocytosis by plaque macrophages, and the enhanced permeability and retention (EPR) effect arising from endothelial dysfunction. This would also provide a platform for potential targeting of the probe to vulnerable plaque-specific proteins such as CX3CL1 [12-16], VCAM-1 [17-19], VEGF [20], or av 33 integrin [21] through antibodies, which require long-circulation to be effective targeting moieties. In addition to an imaging platform, targeting the probe to chemokines such as CX3CL1, CCR2 or CCL5 would have potential therapeutic benefits. These chemokines are all associated with vulnerable plaque, and blocking their expression has been shown to lead to plaque stabilisation and regression $[15,22]$. Developing a long-circulating probe targeted to one or multiple of these proteins would aid in the detection of vulnerable plaque, as well as treating it in situ and improving patient outcomes. 
In order to ensure the long circulation of the probe, the clearance route was of primary consideration. There is a size window between $6-200$ $\mathrm{nm}$ for avoiding renal clearance $(<6 \mathrm{~nm})$ [23] and clearance immediately through the reticuloendothelial system $(>200 \mathrm{~nm})$. Core size was measured by transmission electron microscopy (TEM) (Figure 1) and hydrodynamic size through dynamic light scattering (DLS) (Table 1). Surface charge particularly affects interaction with the immune system, where neutral agents are longer-circulating, positively-charged agents clear faster due to higher intracellular uptake resulting from the electrostatic attraction to the cell membrane, and opsonisation by proteins in the blood stream accelerating phagocytosis [24-26]. Negatively-charged agents are not as long-circulating as neutral agents but are better than positively-charged agents for antibody targeting.

DLS and visual inspection both showed the formation of large aggregates with both amine-based surface ligands, and PEI in particular, was very challenging to exchange onto the nanoparticles despite testing multiple conditions. PMAO showed much narrower size dispersions and improved stability, in water and phosphate buffered saline in accordance with literature findings [27]. The carboxylic acid groups on the surface of the PMAOcoated particles resulted in a negative surface charge, and that in combination with the narrower size dispersion, lack of aggregation and long-term stability made it the most suitable choice for this contrast agent. PMAO has also not seen application in vivo yet, and the probe is therefore novel in surface functionalisation, and looks to be a promising platform for many applications. Nanoparticle contrast agents are easily tuned to many different targets and applications, and - $\mathrm{COOH}$ groups are easily functionalised with targeting moieties for molecular imaging, dyes or fluorophores for optical imaging and potentially histology, chelators for radionuclides or gadolinium, meaning that this probe has potential across a wide spectrum of applications and modalities.

The relaxivity measurements were the decisive factor in selecting the lead-candidate for antibodycoupling, with the $10 \mathrm{~nm}$ nanoparticle cores showing the highest $\mathrm{r}_{2}\left(18.806 \mathrm{mmol}^{-1} \mathrm{~s}^{-1}\right)$. An anti-CX3CL1 antibody was coupled to the surface of the probe through carbodiimide coupling to test the feasibility of molecular targeting, and tests indicated that the antibody was successfully coupled to the nanoparticle surface, and that it retained binding ability after coupling.

\section{In vitro evaluation}

A cell viability assay using RAW 264.7 murine macrophages was undertaken to confirm the contrast agent was non-toxic. Cells were incubated with varying concentrations of iron in excess of what might be encountered in vivo, and the SPIONs were compared to $\mathrm{FeCl}_{3}$. Cell viability was assessed using a standard tetrazolium/formazan cell viability dye [2,3-bis-(2-methoxy, 4-nitro, 5-(sulfophenyl)-2Htetrazolium-5-carboxanilide) (XTT)] measured with absorbance spectroscopy, by minor modification of previous methods [28-32]. Higher measured cell survival was observed for those cells treated with SPIONs as opposed to $\mathrm{FeCl}_{3}$ at all concentrations, as can be observed in Figure 2. For each concentration, cell survival was higher for SPIONs than the corresponding amount of $\mathrm{FeCl}_{3}$, further evidence that the SPIONs were at least as well tolerated as unencapsulated Fe. For reference, Fe is measurable in peripheral serum at a peak concentration of approximately $10 \mathrm{mM}$ after a conventional dietary iron supplement [33] (Figure 2).
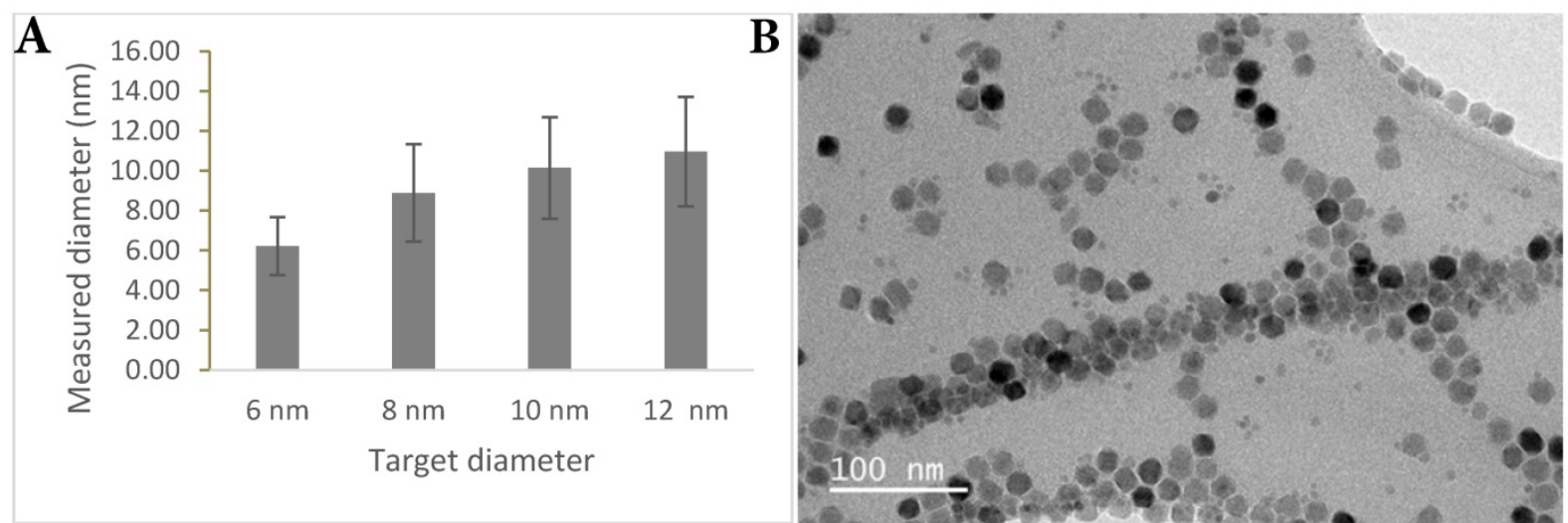

Figure 1. TEM characterisation. (A) Graph showing measured nanoparticle core size versus projected nanoparticle core size. (B) TEM image showing irregular faceting of nanoparticle cores. 
A

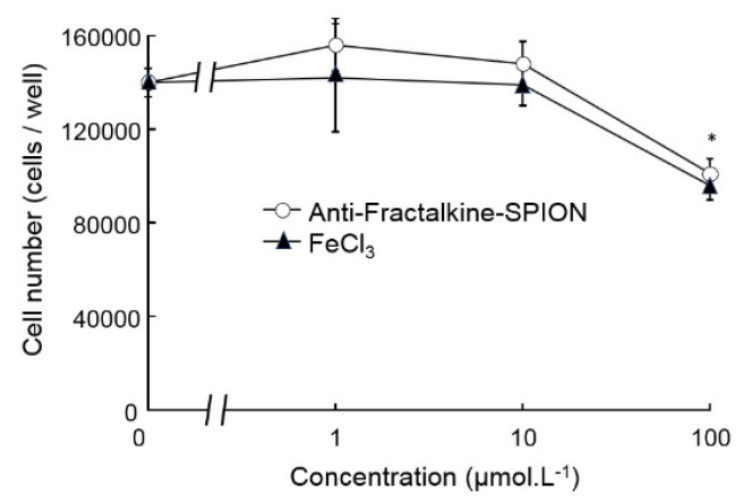

B

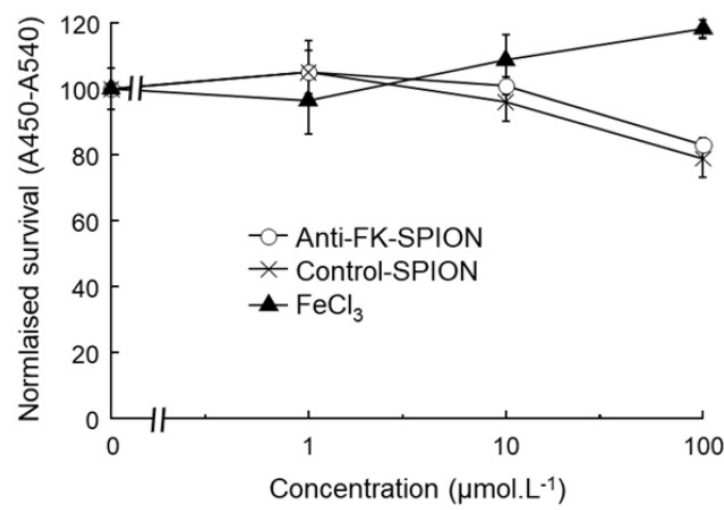

Figure 2. Cell survival assays with SPIONS. Graphs demonstrating measured cell survival of RAW 264.7 murine macrophages at 24 hours incubation with varying concentrations of iron in the form of anti-Fractalkine SPIONs or $\mathrm{FeCl}_{3}$. (A) Cell counts using a hemacytometer at 24 hours showing an equivalent and slight loss of cells at 24 hours at extreme concentrations $(100 \mu \mathrm{M})$ of SPIONs and $\mathrm{FeCl}_{3}$. (B) Colorimetric cell survival assay based on formazan (XTT) reduction, also carried out at 24 hours and also showing an equivalent slight loss of cells at 24 hours at extreme concentrations (100 $\mu$ M) of SPIONs, whether targeted or untargeted (no antibody). $\mathrm{FeCl}_{3}$ increases apparent cell number, although that is likely to represent an artefact e.g. mitochondrial dysfunction. Data were also obtained at 2 hours, when there was very little effect (not shown).

Table 1. Hydrodynamic size and surface potential measurements for all synthesised nanoparticles

\begin{tabular}{lll}
\hline Sample & Diameter in $\mathrm{nm}$ & Potential in $\mathrm{mV}$ \\
\hline $6 \mathrm{~nm}$ iron oxide with PEI & 148.5 & 3.30 \\
$8 \mathrm{~nm}$ iron oxide with PEI & 206.5 & 1.60 \\
$10 \mathrm{~nm}$ iron oxide with PEI & --- & 10.91 \\
$12 \mathrm{~nm}$ iron oxide with PEI & 371.5 & 5.73 \\
$6 \mathrm{~nm}$ iron oxide with PMAO & 113.2 & -42.11 \\
$8 \mathrm{~nm}$ iron oxide with PMAO & 104.7 & -36.66 \\
$10 \mathrm{~nm}$ iron oxide with PMAO & 194.6 & -36.21 \\
$12 \mathrm{~nm}$ iron oxide with PMAO & 139.6 & -22.84 \\
$6 \mathrm{~nm}$ iron oxide with alendronate & 199.5 & 26.86 \\
$8 \mathrm{~nm}$ iron oxide with alendronate & 292.7 & 12.12 \\
$10 \mathrm{~nm}$ iron oxide with alendronate & 194.0 & 26.60 \\
$12 \mathrm{~nm}$ iron oxide with alendronate & 266.4 & 28.44 \\
\hline
\end{tabular}

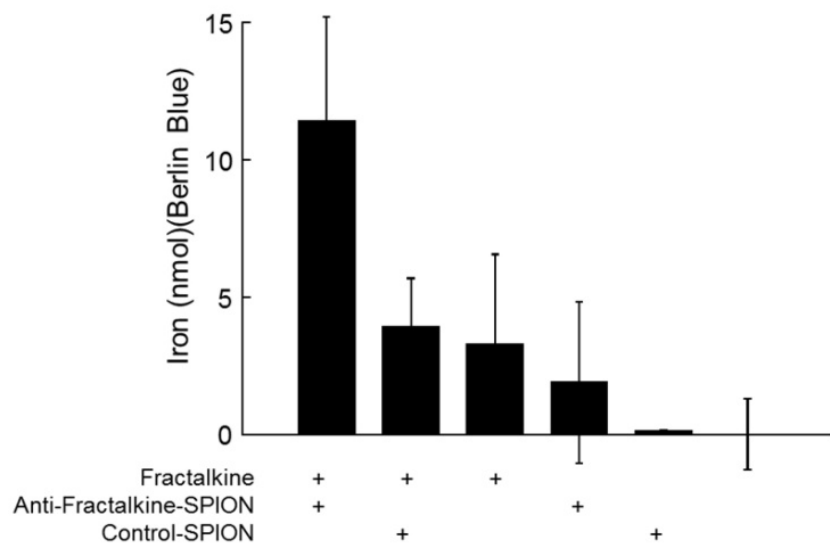

Figure 3. Specific binding of anti-factalkine SPIONs to immobilised fractalkine shown by Berlin-Blue based iron measurement. Graph showing bound as detected by Berlin Blue and UV-Vis. spectroscopy in wells coated with CX3CL1 and incubated with targeted and non-targeted SPIONs. $*_{p}<0.05$, ANOVA.

In order to confirm that the anti-fractalkineSPION conjugate specifically targeted fractalkine, a binding assay was designed. Both targeted and non-targeted SPIONs were incubated against murine CX3CL1 or control-coatings on 96-well plates (see Methods). To obtain an unambiguous demonstration that iron-containing particles were captured by plateadsorbed fractalkine, unbound particles were removed through a gentle wash and plate-bound iron detected a reaction product with Berlin Blue (acidified potassium ferricyanide, a classical reagent for detecting immobilized iron) added. After 10-20 mins incubation, Berlin Blue/iron complex was measured by UV-Vis. spectroscopy. The results (Figure 3) demonstrated higher levels of bound iron detected in the wells with targeted SPIONs as opposed to control SPIONs.

\section{In vivo evaluation}

The contrast agent was tested in a murine model of advanced (vulnerable) plaque: $6 \mathrm{ApoE}^{-/} \mathrm{C} 57 / \mathrm{Bl} 6$ female mice fed a high fat diet with a cuff placed on the left carotid artery for 9 weeks to cause a stenosis and changes in wall shear stress patterns. The model was developed to cause a vulnerable plaque upstream of the cuff and a stable plaque downstream of the cuff, which made it particularly suitable for testing this contrast agent by enabling the direct comparison of uptake in known vulnerable and stable plaque sites [34].

The mice were first injected with a gadolinium-containing elastin-specific MR contrast agent (Gd-ESMA) which has been characterised in atherosclerosis models, allowed to visualise the location and extend of plaque and provided a benchmark for the novel contrast agent. The mice were then injected with the SPIONs and imaged 24 hours post-injection. The results are illustrated in Figures 4 and 5. 
A
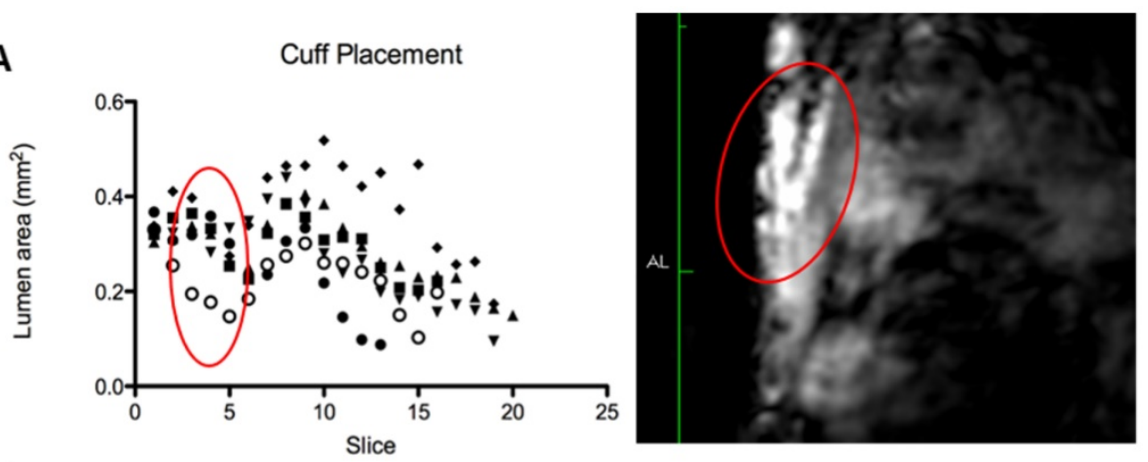

B
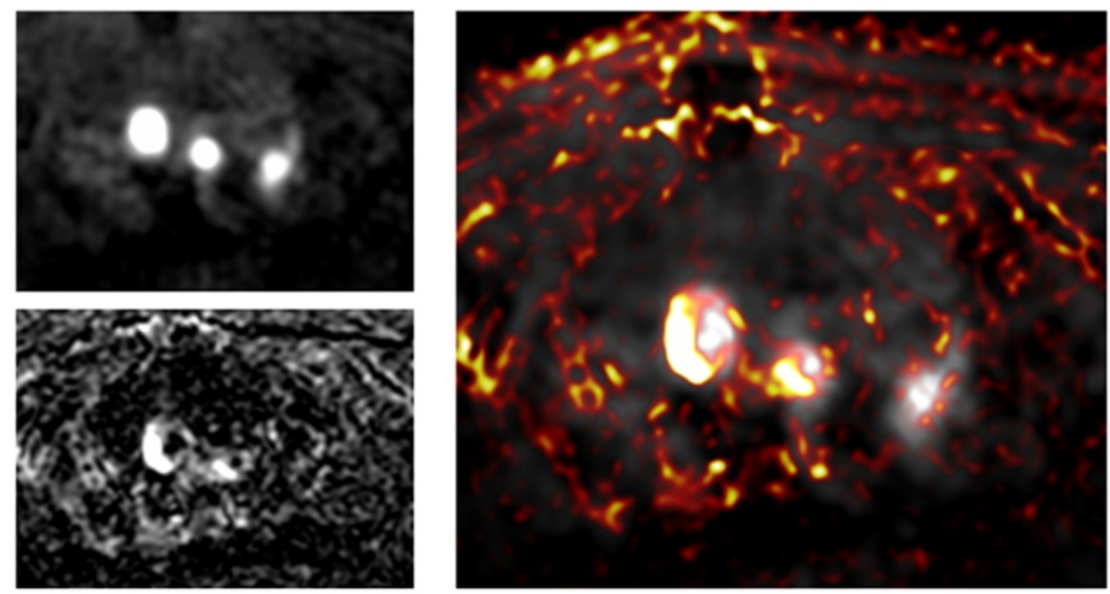

C
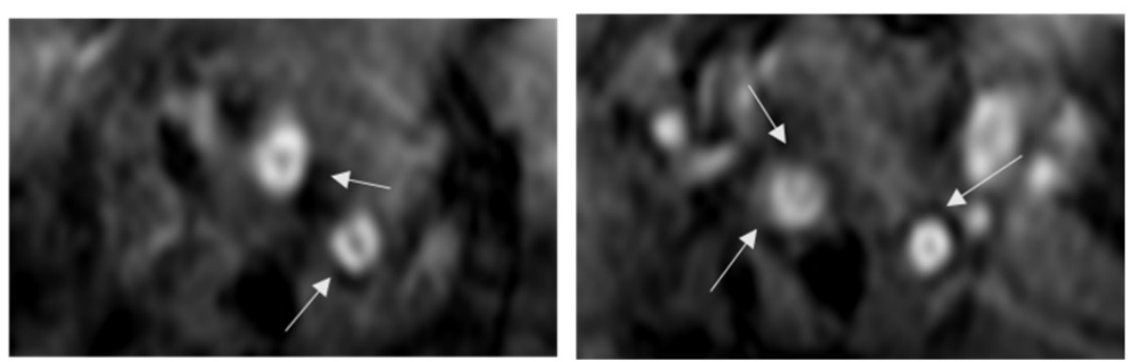

Figure 4. Top to bottom, (A) Left: graph showing lumen area versus slice number, demonstrating a consistent decrease in lumen area from slice 3-5 (standardised relative to aortic arch) in all 6 animals (represented by different legends) corresponding to cuff location; right: anatomical scan showing the location of the cuff and resulting arterial stenosis; (B) Left: Magnetic Resonance Angiography scan (Top) showing the brachiocephalic artery, left carotid artery, and left subclavian artery (L-R), Bottom: Inversion recovery image showing plaque areas with Late Gadolinium enhancement from Gd-ESMA in the vessel walls. Right: Image overlay showing the areas of enhancement in relation to the vessel lumen. (C) Two examples of $\mathrm{T}_{2}{ }^{*}$ echo scans based on the protocol from Makowski et. al. [35] showing negative contrast areas in the vessel walls.

The Gd-ESMA scans showed a significant difference between uptake in the cuffed vessel compared with the contralateral control vessel $(p=$ 0.018), demonstrating that the cuff placement and hyperlipidemia led to the formation of plaque more rapidly than hyperlipidemia alone through alterations of wall shear stress. There was also a significant difference between the Gd-ESMA signal in the cuffed disease vessel compared to both the left and right carotid arteries in the control animals $((p=0.002$, 0.000095), supporting the plaque formation as a result of hyperlipidemia and placement of the cuff with resultant stenosis (Figure 5). The data was analysed using multiple non-parametric t-tests corrected for multiple comparisons by the Holm-Sidak method, and was found to be statistically significant. There was no notable difference in Gd-ESMA signal between the stable and vulnerable plaque regions measured in three slices above and below the stenosis of the cuff, but it was not expected that elastin content would differ significantly, particularly given that Gd-ESMA binds to both the cross-linked mature form of elastin, and its precursor tropoelastin. Interestingly, the brachiocephalic artery was observed to be enlarged related to other murine atherosclerosis models, and this is likely to be a result of compensation due to the restriction the cuff places on the left carotid. 
A Late Gadolinium-Enhancement after Gd-ESMA

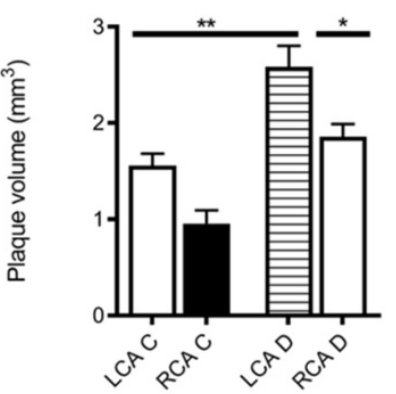

B

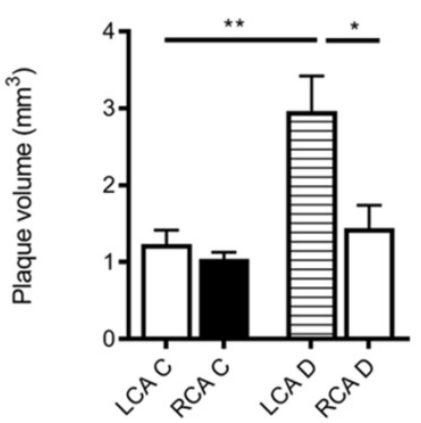

C

Pre- and Post-cuff

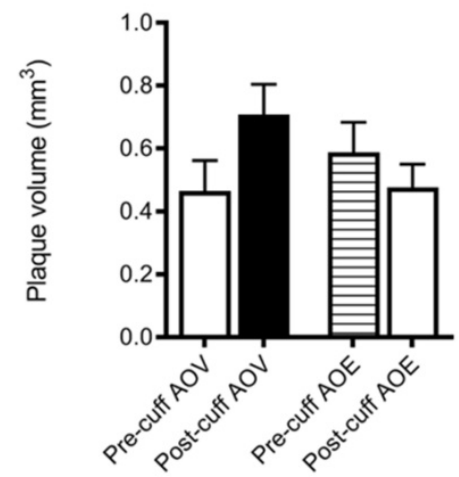

Figure 5. (A) Graph showing plaque volume as measured by area of late-gadolinium enhancement in both the left (LCA) and right (RCA) carotid arteries of control (left) and diseased (right) animals, showing that cuff placement and hyperlipidemia increase plaque size in the instrumented diseased left carotid artery $(* p<0.05$, student's t-test); (B) graph showing plaque volume as measured by T2* enhancement in the left (LCA) and right (RCA) carotid arteries of control (left) and diseased (right) animals, showing that cuff placement hyperlipidemia increase plaque size in the instrumented diseased left carotid artery (*p<0.05, student's t-test); (C) graph showing area of void (AOV) arising from SPIONs and area of late-gadolinium enhancement (AOE) arising from Gd-ESMA in control regions pre- (upstream) and post-cuff (downstream), although differences are present, no level of significance was reached.

The SPION behavior mirrored Gd-ESMA signal, validating the novel contrast agent against an established agent to measure plaque burden. Again, there was a significant difference between the modified vessel and the internal control vessel $(p=$ 0.018 ), and between the modified, diseased vessel and both vessels in the control animalsp $=0.005,0.002$ ) (Figure 5). The data was analysed using multiple nonparametric t-tests corrected for multiple comparisons by the Holm-Sidak method, and was found to be statistically significant.

There was no significant difference between the uptake of either contrast agent between the vulnerable plaque upstream of the cuff, and the stable plaque downstream of the cuff $(\mathrm{p}=0.32$, student's t-test) (Figure 5).

\section{Histological verification}

Imaged plaques and control sections of artery were then analysed through histology and immunohistochemistry, staining sections for elastin, tropoelastin, CD68, CX3CL1 (a marker for vulnerable plaque), and iron. Berlin blue staining for iron confirmed the presence of SPION-derived iron in the plaques of diseased vessels (Figure 8), but there was no Berlin blue staining in the control vessels. There was a significant difference in expression of both CD68 which is a macrophage marker, and Fractalkine between sections of tissue from diseased and healthy control animals. Levels of tropoelastin were also elevated in plaque regions. These findings are illustrated in Figures 6 and 7.

\section{Conclusions}

In conclusion, this paper illustrates the development of a novel superparamagnetic iron oxide nanoparticle contrast agent which successfully allowed imaging of atherosclerotic plaques with MRI. Although the probe is not specific for vulnerable plaque, it is non-toxic and accumulates in similar regions to an elastin-targeted agent for visualizing plaque burden. It also provides a good platform for further refinement towards a vulnerable plaquespecific contrast agent in the future. The novel probe has successfully been labelled with an antibody, and the conjugated antibody has been demonstrated to retain binding specificity for the target. There is a limitation in that the nanoparticles were labelled with an anti-CX3CL1 antibody, however this labelling was at a low proportion of the injected dose and so the 
probe has not been treated as a targeted agent. Because of this, no conclusions can be drawn regarding potential levels of CX3CL1 expression in the plaques, although previous work by Cheng et. al. suggests that CX3CL1 is elevated in vulnerable plaques [11].

\section{Methods}

\section{Chemistry}

Synthesis of $6 \mathrm{~nm}$ hydrophobic SPIONs [36]

$\mathrm{Fe}(\mathrm{acac})_{3} \quad(0.71 \mathrm{~g}, 2 \mathrm{mmol})$ and 1,2-hexadecanediol $(2.58 \mathrm{~g}, 10 \mathrm{mmol})$ were dissolved in benzyl ether $(20 \mathrm{ml})$. Oleic acid $(2.11 \mathrm{ml}, 6 \mathrm{mmol})$ and oleylamine $(2.80 \mathrm{ml}, 6 \mathrm{mmol})$ were added, and the mixture was stirred for 2 hours under $\mathrm{N}_{2}$ at $200{ }^{\circ} \mathrm{C}$. The mixture was then heated to reflux $\left(300^{\circ} \mathrm{C}\right)$ for 1 hour and cooled by the removal of the heat source.

Nanoparticles were precipitated with EtOH (40 $\mathrm{ml}$ ) and centrifuged for 30 minutes at $4000 \mathrm{rpm}$. Pellet was re-dispersed in hexane $(20 \mathrm{ml})$, centrifuged for 10 minutes at $4000 \mathrm{rpm}$ and then precipitated again with EtOH $(20 \mathrm{ml})$. The solution was centrifuged for 30 minutes at $4000 \mathrm{rpm}$, supernatant removed and pellet dried under vacuum. SPIONs were stored dry.

\section{Seed-mediated growth of SPIONs [36]}

$\mathrm{Fe}(\mathrm{acac})_{3} \quad\left(\begin{array}{llll}0.18 & \mathrm{~g}, & 0.5 & \mathrm{mmol})\end{array}\right.$ and 1,2-hexadecanediol $(0.65 \mathrm{~g}, 2.5 \mathrm{mmol})$ were dissolved in benzyl ether $(15 \mathrm{ml})$. Oleic acid $(0.70 \mathrm{ml}, 0.5 \mathrm{mmol})$, oleylamine $(0.93 \mathrm{ml}, 0.5 \mathrm{mmol})$ and SPIONs $(5-10 \mathrm{mg})$ were added and the mixture was stirred for 1 hour at $200^{\circ} \mathrm{C}$. The mixture was then heated to reflux $\left(300^{\circ} \mathrm{C}\right)$ for half an hour and cooled by the removal of the heat source.

The workup was performed as with $6 \mathrm{~nm}$ hydrophobic SPIONs.

\section{Coating with poly(maleic anhydride-alt-1-octadecene)} [10]

Poly(maleic anhydride-alt-1-octadecene) (PMAO) $(45 \mathrm{mg})$ was dissolved in $\mathrm{CHCl}_{3}(20 \mathrm{ml})$. The SPIONs $(2 \mathrm{mg})$ were added and mixture was stirred for 30 minutes. Chloroform was removed, and residue re-dissolved in a small amount of $\mathrm{CHCl}_{3}$. NaOH (aq) $(0.05 \mathrm{M})$ was added and mixture was heated at $60^{\circ} \mathrm{C}$ until SPIONs transferred to the aqueous phase.
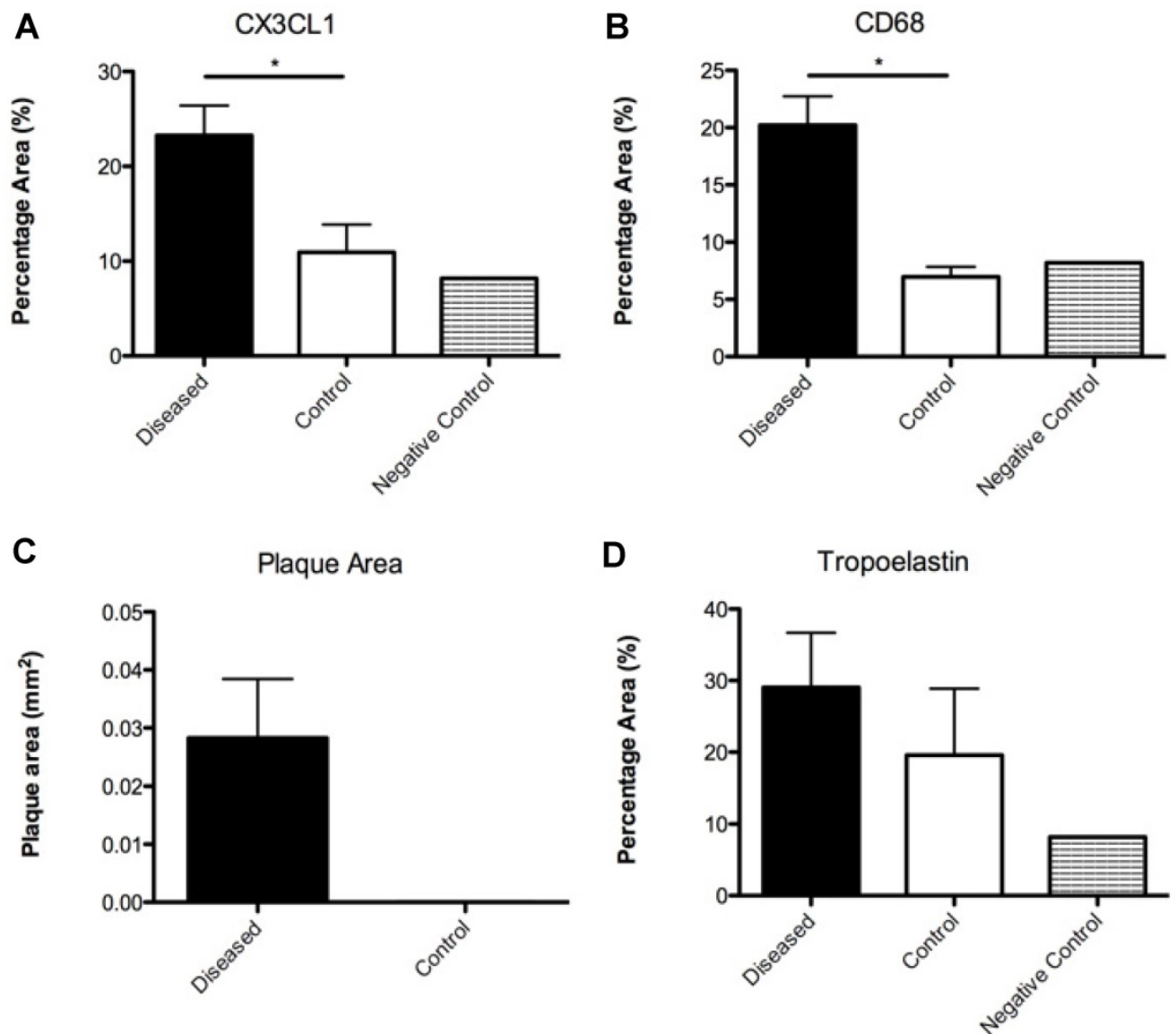

Figure 6. From top to bottom and left to right: (A) Graph illustrating the percentage vessel wall area of CX3CLI measured in diseased and control sections; (B) Graph illustrating the percentage vessel wall area of CD68 measured in diseased and control sections; (C) Graph illustrating the plaque area measured in diseased and control sections; (D) Graph illustrating the percentage vessel wall area of tropoelastin measured in diseased and control sections. 
A

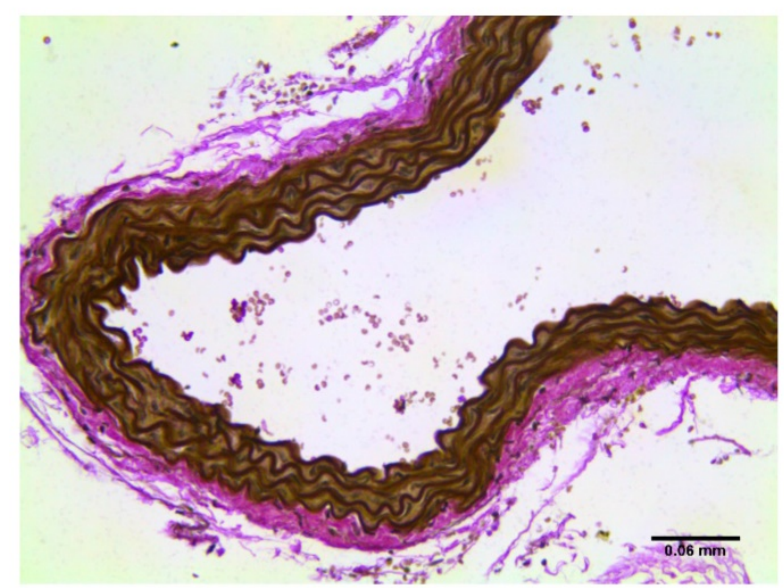

C

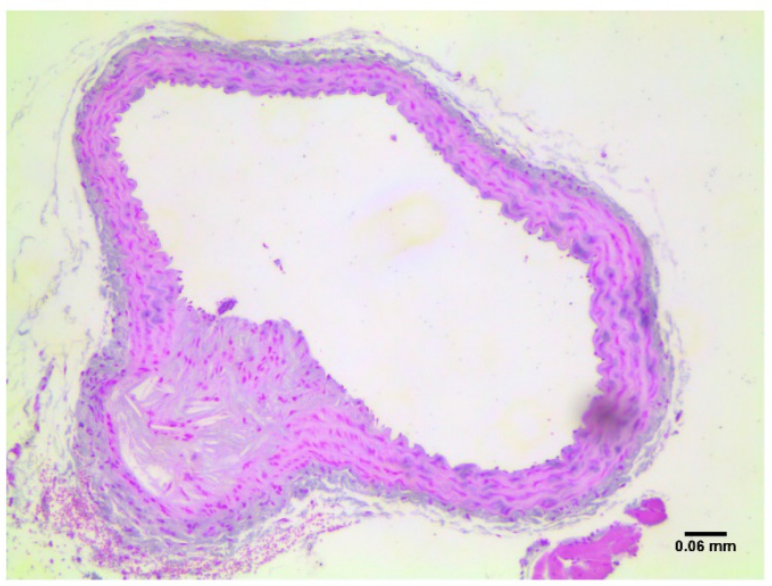

E

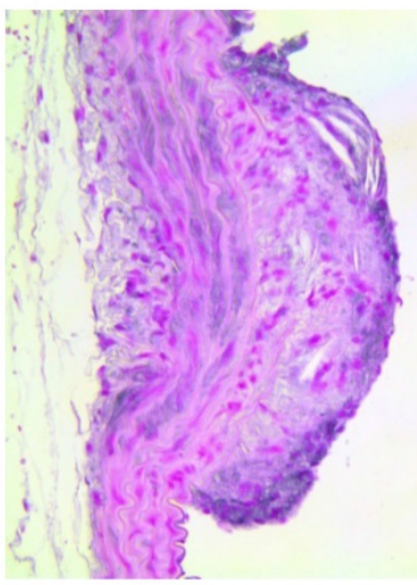

B

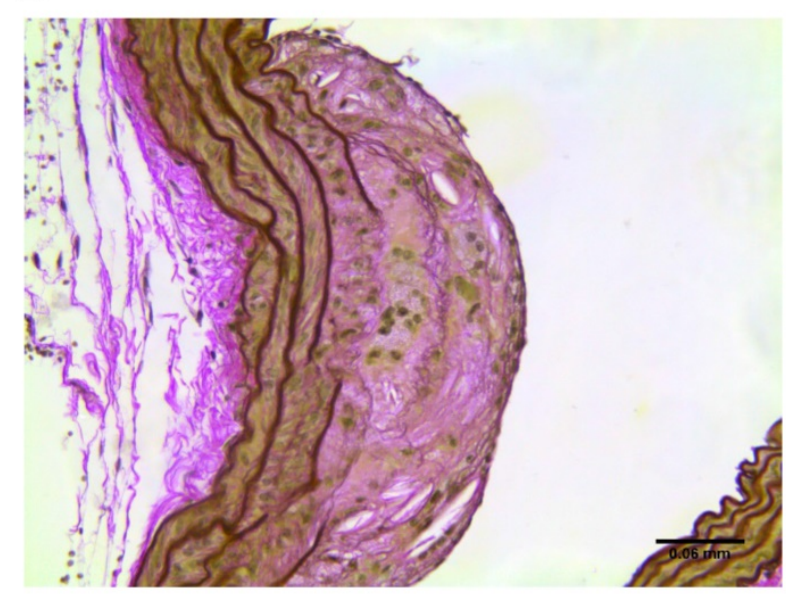

D

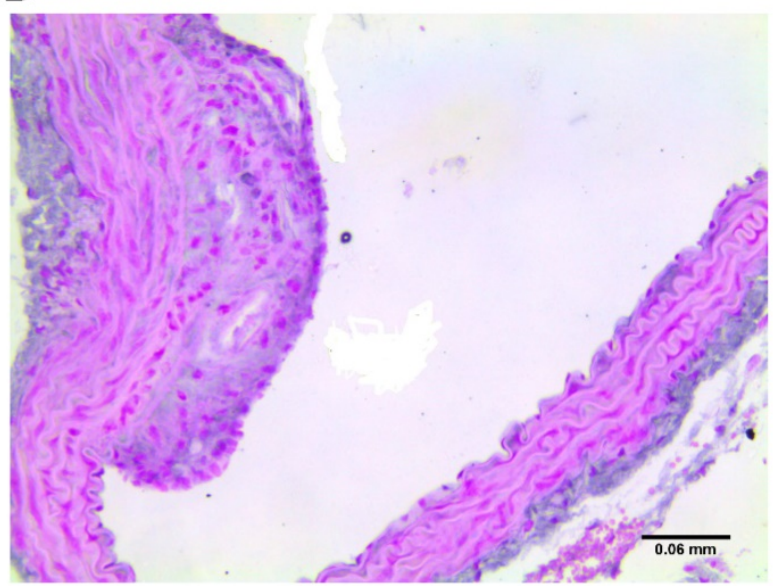

$\mathbf{F}$

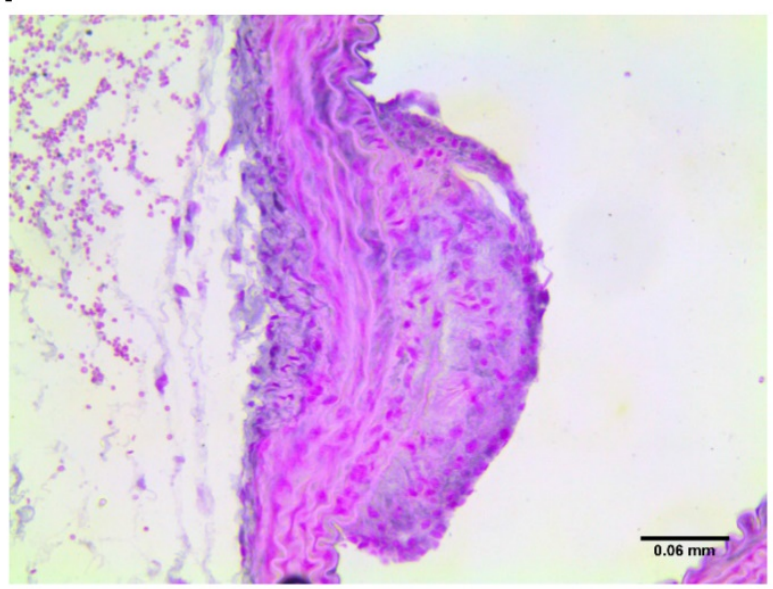

Figure 7. (A) Section of control vessel stained for elastin and, (B) section of diseased vessel stained for elastin showing a plaque. (C-D) Sections of diseased vessel stained for tropoelastin, showing a plaque. (E) Section of diseased vessel showing a plaque stained for CD68, and (F) section of diseased vessel showing a plaque stained for CX3CLI.

The solution was filtered using on a $0.45 \mu \mathrm{m}$ filter, and then concentrated by centrifugation with a filter of 30k Mw. Particles were re-suspended in water and stored.

\section{Coating with alendronate [11]}

Sodium alendronate trihydrate $(50 \mathrm{mg})$ was dissolved in water $(5 \mathrm{ml})$ adjusted to $\mathrm{pH} 9$ with $1 \mathrm{M}$ $\mathrm{KOH}$. The SPIONs $(6 \mathrm{mg})$ were suspended in THF. The solutions were combined and stirred at room temperature for 2 days. The solution was magnetically decanted and washed with acetone $(3 \times 10 \mathrm{ml})$. The particles were re-suspended in water and stored. 


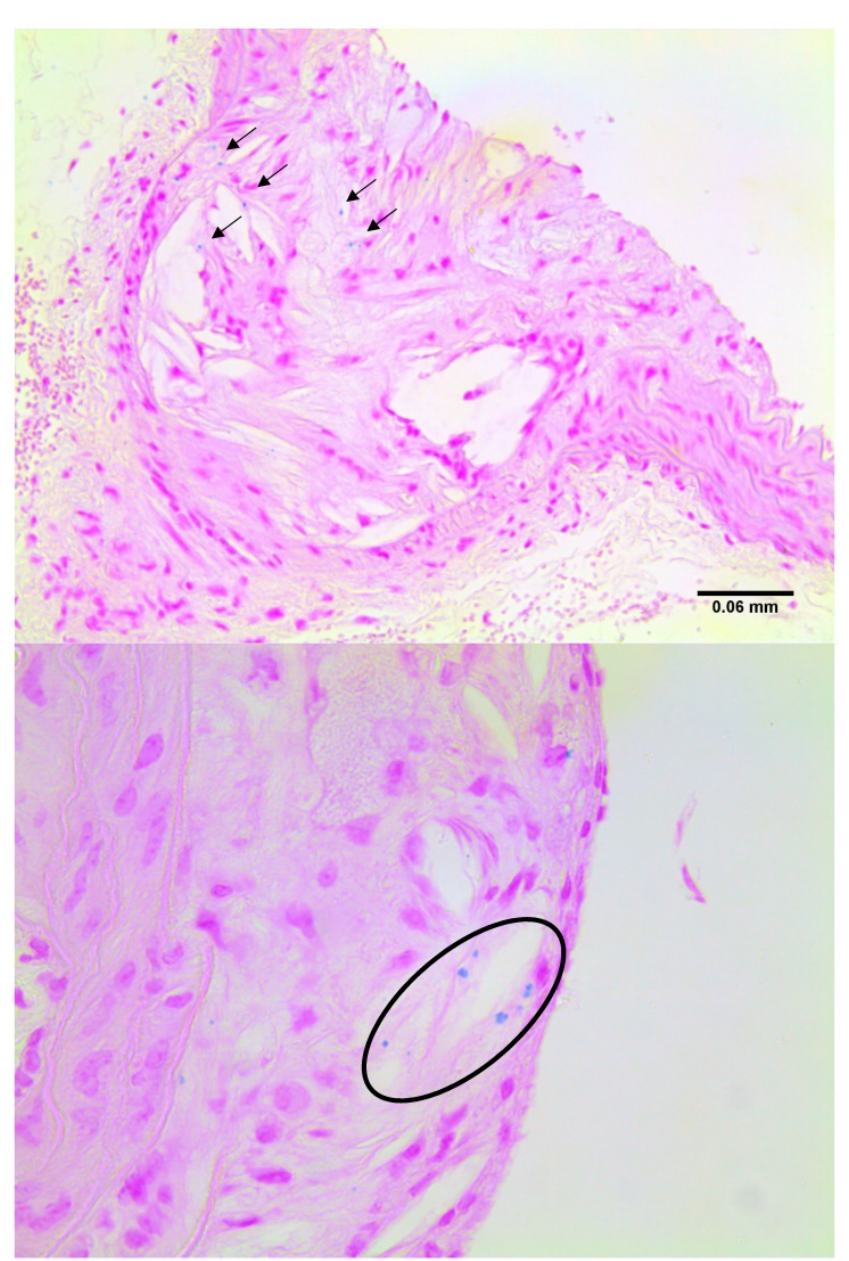

Figure 8. Examples of sections stained for iron with Berlin Blue, highlighting the blue deposits in plaque regions.

\section{Biology}

In vitro cell toxicity assay

\section{Preparation of XTT working reagent:}

$5 \mathrm{ml}$ of PBS (Phosphate Buffered Saline) and 50 $\mu \mathrm{l}$ of XTT $(25 \mathrm{mg} / \mathrm{ml}$ ) and $0.5 \mu \mathrm{l}$ PMS (Phenazine methosulfate, $50 \mathrm{mg} / \mathrm{ml}$ ) was added into a clean $15 \mathrm{ml}$ conical centrifuge tube.

In a 96 well plate each well was seeded with 200 $\mu \mathrm{l}$ of $10 \mathrm{k}$ RAW 264.7 cells in IMDM with $10 \%$ FBS. The plate was incubated overnight, and then treated with SPIONs at different concentrations: $0 \mu \mathrm{M}, 1 \mu \mathrm{M}, 10$ $\mu \mathrm{M}$ and $100 \mu \mathrm{M}$. The plate was incubated overnight, and $20 \mu \mathrm{l}$ of XTT reagent as prepared above was added to the wells and incubated. The absorbance was measured at 2 and 24 hours post-addition of XTT at 450 and $540 \mathrm{~nm}$.

\section{Animal Model}

All animal experiments complied with the Animals (Scientific Procedures) Act 1986 and were carried out under PPL 70/8482 at King's College London.

Homozygous female ApoE-/- mice (C57BL/6J background) were acquired from Charles Rivers Laboratories (Edinburgh, UK) and bred within the Behavioural Sciences Unit of the Rayne Institute. The housing and care of the animals, and all procedures in this study were performed in accordance with the guidelines and regulations of the UK home office.

At 11 weeks of age the mice were placed on high fat diet (HFD) (21\% fat, $0.25 \%$ cholesterol wt/wt from LBS-Biotech) for two weeks prior to surgery. Two weeks after commencement of HFD the mice underwent surgery for the placement of a rigid polyether ketone perivascular cuff (Promolding, The Netherlands) around the left carotid. The cuff was supplied in two pieces and when assembled the lumen narrowed from $500 \mu \mathrm{m}$ to $250 \mu \mathrm{m}$ over $1.5 \mathrm{~mm}$. The animals were kept under anaesthesia at 1.5-2.5\% isoflurane and $2 \% \mathrm{O}_{2}$, with anaesthetic depth being assessed from respiratory rate, hind limb muscle tone, and pedal withdrawal reflex as previously described [34].

The animals then remained on HFD for a further 9 weeks before scanning and sacrifice. Animals were euthanised under Schedule 1 with an overdose of anaesthetic followed by exsanguination and flushing of the vasculature with saline.

Healthy ApoE-/- mice with no cuff fed a normal chow diet were used as controls.

\section{Imaging Studies: Gd-ESMA}

Based on previous studies carried out by Makowski et. al. [37] animals were injected with 0.2 $\mathrm{mmol} / \mathrm{kg}$ Gd-ESMA in $150 \mu \mathrm{l}$ sterile saline through the tail vein. The animals were then scanned 1 hour post-injection.

In vivo vessel wall imaging was performed using a Philips Achieva MR scanner (Philips Healthcare, Best, The Netherlands) equipped with a clinical gradient system $\left(30 \mathrm{mT} \mathrm{m}^{-1}, 200 \mathrm{mT} \mathrm{m}^{-1} \mathrm{~ms}^{-1}\right)$.

The brachiocephalic artery (BCA) and left and right carotids were imaged using a single-loop surface coil (diameter $=23 \mathrm{~mm}$ ). Mice were placed in prone position after intravenous administration of 0.2 $\mathrm{mmol} / \mathrm{kg}$ Gd-ESMA (Lantheus Medical Imaging, North Billerica, MA). Anesthesia was induced with $5 \%$ and maintained with $1 \%$ to $2 \%$ isoflurane during the MRI experiments.

After a 3-dimensional gradient recalled echo scout scan, contrast-enhanced angiography images were acquired with a field of view $(F O V)=30 \times 30 \times 8$ $\mathrm{mm}$, matrix $=200 \times 200$, in-plane resolution $=0.15 \times$ $0.15 \times 0.5 \mathrm{~mm}$ (reconstructed $=0.10 \times 0.10 \mathrm{~mm}$ ), repetition time $/$ echo time $=15 / 6.1 \mathrm{~ms}$, and flip angle 
$=40^{\circ}$. The maximum intensity projection images were used to plan the subsequent delayed-enhancement (DE) and $\mathrm{T}_{1}$ mapping scans. A 2-dimensional Look-Locker sequence was acquired using the following parameters: $\mathrm{FOV}=30 \times 30 \mathrm{~mm}$, matrix $=$ $80 \times 80$, in-plane resolution $=0.38 \times 0.38 \times 2 \mathrm{~mm}$, repetition time/echo time $=19 / 8.6 \mathrm{~ms}$, repetition time between subsequent inversion recovery pulses $=1000$ $\mathrm{ms}$, and flip angle $=10^{\circ}$. An inversion recovery 3-dimensional fast gradient echo sequence was acquired with $\mathrm{FOV}=30 \times 30 \times 8 \mathrm{~mm}$, matrix $=304 \times$ 304 , in-plane resolution $=0.1 \times 0.1 \times 0.5 \mathrm{~mm}$, repetition time/echo time $=28 / 8 \mathrm{~ms}$, repetition time between subsequent inversion recovery pulses $=1000 \mathrm{~ms}$, and flip angle $=30^{\circ}$. $\mathrm{T}_{1}$ mapping was performed with the same sequence employed for the aorta. The acquisition parameters were as follows: $\mathrm{FOV}=36 \times$ $22 \times 8 \mathrm{~mm}$, matrix $=192 \times 102$, in-plane resolution $=$ $0.18 \times 0.22 \times 0.5 \mathrm{~mm}$, slices $=16$, repetition time $/$ echo time $=9.6 / 4.9 \mathrm{~ms}$, flip angle $=10^{\circ}$.

\section{Imaging Studies: SPIONs}

Animals were injected with $150 \mu \mathrm{l} 6 \mathrm{mM}$ [Fe] SPIONs in sterile saline through the tail vein. The animals were then scanned 24 hours post-injection.

The imaging protocols were based on previous experiments by Makowski et. al. [35] and were carried out on a Phillips 3T Achieva scanner (Philips Healthcare, Best, The Netherlands) equipped with a clinical gradient system $\left(30 \mathrm{mT} \mathrm{m}^{-1}, 200 \mathrm{mT} \mathrm{m}^{-1} \mathrm{~ms}^{-1}\right)$ as before.

The brachiocephalic artery (BCA) and left and right carotids were imaged using a single-loop surface coil (diameter $=23 \mathrm{~mm}$ ). Mice were placed in prone position. Anesthesia was induced with $5 \%$ and maintained with $1 \%$ to $2 \%$ isoflurane during the MRI experiments.

After a 3-dimensional gradient recalled echo scout scan, contrast-enhanced angiography images were acquired with a field of view $($ FOV $)=30 \times 30 \times 8$ $\mathrm{mm}$, matrix $=200 \times 200$, in-plane resolution $=0.15 \times$ $0.15 \times 0.5 \mathrm{~mm}$ (reconstructed $=0.10 \times 0.10 \mathrm{~mm}$ ), repetition time/echo time $=15 / 6.1 \mathrm{~ms}$, and flip angle $=40^{\circ}$. The maximum intensity projection images were

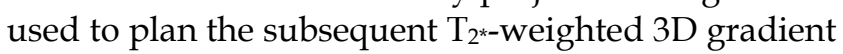
echo scan. ECG-triggering was not used, and a

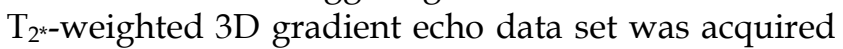
with $\mathrm{FOV}=16 \times 16 \times 8$; matrix $=176 \times 176$; in-plane spatial resolution $=0.12 \times 0.12 \mathrm{~mm}$; slice thickness $=$ $0.5 \mathrm{~mm} ; \mathrm{TR} / \mathrm{TE}=37.88 / 7.32 \mathrm{~ms}$ and flip angle $=25^{\circ}$.

\section{Histology}

\section{Elastin Staining}

Deparaffinised slides were incubated in working elastic stain solution for 10 minutes and rinsed in deionized water. Slides were then placed in ferric chloride solution for 22 seconds, rinsed under running water and checked under the microscope. Slides were rinsed in $96 \%$ ethanol to remove excess iodine, then rinsed in deionized water. Slides were incubated in van Gieson solution for 1-3 minutes. Finally, slides were dehydrated through a gradient of ethanol, cleared in xylene, and mounted.

\section{Prussian Blue Staining}

$20 \%$ aqueous solution of hydrochloric acid (20 $\mathrm{ml}$ conc. $\mathrm{HCl}, 80 \mathrm{ml}$ water) and $10 \%$ potassium ferrocyanide trihydrate $(10 \mathrm{~g}, 100 \mathrm{ml}$ water) were prepared and mixed fresh before staining. Slides were immersed in the solution for 20 minutes then washed in deionized water three times before counterstaining with nuclear fast red for 5 minutes. Finally, slides were dehydrated through a gradient of ethanol, cleared in xylene, and mounted.

\section{Immunohistochemistry}

Deparaffinised slides were stained for tropoelastin, CD68 or $\mathrm{CX} 3 \mathrm{CL} 1$ using the following protocol:

Endogenous peroxidase was inhibited by incubation of the slides in 3\% oxygenated water and methanol for 10 minutes. Slides were then washed in deionized water for 10 minutes. Antigen retrieval was performed by boiling the samples for 2 minutes at 110 ${ }^{\circ} \mathrm{C}$ in citrate buffer in a pressurized antigen retrieval unit and then allowed to cool. Samples were washed with PBS for 3 minutes, and blocking was performed for 1 hour at room temperature with 10\% donkey serum in PBS. Incubation with the primary antibody was carried out overnight at $4{ }^{\circ} \mathrm{C}$ diluted 1:100 in PBS $2 \%$ normal donkey serum. Samples were allowed to temper at room temperature for 1 hour, then slides were washed twice in PBS for 20 minutes. Slides were incubated with anti-rabbit HRP polymer for 45 minutes at room temperature then washed 3 times in PBS for 5 minutes.

Slides were revealed with Vector SG Peroxidase substrate for 10 minutes at room temperature PBS (5 ml), Chromogen (75 $\mu \mathrm{l})$, Hydrogen peroxide $(120 \mu \mathrm{l})$.

Samples were washed twice in PBS for 5 minutes, before counterstaining with nuclear fast red for 5 minutes. Slides were washed with deionized water then finally dehydrated through a gradient of ethanol, cleared in xylene, and mounted.

\section{Supplementary Material}

Supplementary figure S1.

http://www.ntno.org/v04p0184s1.pdf 


\section{Acknowledgements}

We thank Dr. Javier Hernández-Gil for assistance in nanoparticle synthesis, Mahmoud Ardakani and Ecaterina Ware for TEM characterisation and evaluation, and Patricia Carry for assistance with ICP measurements. This work was funded via the King's College London \& Imperial College London, EPSRC Centre for Doctoral Training in Medical Imaging (EP/L015226/1). NJL is grateful for a Royal Society Research Merit award. JJB is funded by the British Heart Foundation (FS/13/12/30037).

\section{Competing Interests}

The authors have declared that no competing interest exists.

\section{References}

1. Gulani V, Calamante F, Shellock FG, Kanal E, Reeder SB. Gadolinium deposition in the brain: summary of evidence and recommendations. Lancet Neurol. 2017;16(7):564-570. doi:10.1016/S1474-4422(17)30158-8.

2. World Health Organisation. WHO | Top 10 causes of death https://www.who.int/news-room/fact-sheets/detail/the-top-10-causes-ofdeath. Published 2018. Accessed April 8, 2019.

3. British Heart Foundation. BHF CVD STATISTICS COMPENDIUM 2017. 2017.

4. Bakhshi H, Meyghani Z, Kishi S, et al. Comparative Effectiveness of CT-Derived Atherosclerotic Plaque Metrics for Predicting Myocardial Ischemia. JACC Cardiovasc Imaging. 2019;12(7):1367-1376. doi:10.1016/ j.jcmg.2018.05.019.

5. Shen ZT, Zheng S, Gounis MJ, Sigalov AB. Diagnostic Magnetic Resonance Imaging of Atherosclerosis in Apolipoprotein E Knockout Mouse Model Using Macrophage-Targeted Gadolinium-Containing Synthetic Lipopeptide Nanoparticles. PLoS One. 2015;10(11):e0143453. doi:10.1371/journal.pone. 0143453.

6. Pan D, Caruthers SD, Senpan A, Schmieder AH, Wickline SA, Lanza GM. Revisiting an old friend: manganese-based MRI contrast agents. Wiley Interdiscip Rev Nanomedicine Nanobiotechnology. 2011;3(2):162-173. doi:10.1016/j.molcel.2007.05.041.A.

7. Usman A, Patterson AJ, Sadat U, Tang TY, Graves MJ, Gillard JH. Assessment of Carotid Plaque Inflammation in Diabetic and Nondiabetic Patients - An Exploratory Ultrasmall Superparamagnetic Iron Oxide-Enhanced Magnetic Resonance Imaging Study. J Stroke Cerebrovasc Dis. 2017;26(4):858-862. doi:10.1016/j.jstrokecerebrovasdis.2016.10.034.

8. Smits LP, Tiessens F, Zheng KH, Stroes ES, Nederveen AJ, Coolen BF. Evaluation of ultrasmall superparamagnetic iron-oxide (USPIO) enhanced MRI with ferumoxytol to quantify arterial wall inflammation. Atherosclerosis. 2017;263:211-218. doi:10.1016/j.atherosclerosis.2017.06.020.

9. Sun S, Zeng H, Robinson DB, et al. Monodisperse MFe2O4 (M= Fe, Co, Mn) Nanoparticles. J Am Chem Soc. 2004·126(1):273-279. doi:10.1149/1.3365025

10. Moros M, Pelaz B, López-Larrubia P, García-Martin ML, Grazú V, de la Fuente JM. Engineering biofunctional magnetic nanoparticles for biotechnological applications. Nanoscale. 2010;2(9):1746-1755. doi:10.1039/c0nr00104j.

11. Keasberry NA, Bañobre-López M, Wood C, Stasiuk GJ, Gallo J, Long NJ. Tuning the relaxation rates of dual-mode $\mathrm{T} 1 / \mathrm{T} 2$ nanoparticle contrast agents: a study into the ideal system. Nanoscale. 2015;7:16119-16128. doi:10.1039/C5NR04400F

12. Cheng C, Tempel D, van Haperen $R$, et al. Shear stress-induced changes in atherosclerotic plaque composition are modulated by chemokines. J Clin Invest. 2007:117(3):616-626 doi:10.1172/JCI28180.

13. Zhang X, Feng X, Cai W, et al. Chemokine CX3CL1 and its receptor CX3CR1 are associated with human atherosclerotic lesion volnerability. Thromb Res. 2015;135(6):1147-1153. doi:10.1016/j.thromres.2015.03.020.

14. Flierl U, Bauersachs J, Schäfer A. Modulation of platelet and monocyte function by the chemokine fractalkine ( $\mathrm{CX}<\mathrm{inf}>3</$ inf $>C L 1)$ in cardiovascular disease. Eur J Clin Invest. 2015;45(6):624-633. doi:10.1111/eci.12443.

15. Zernecke A, Weber C. Chemokines in the vascular inflammatory response of atherosclerosis. Cardiovasc Res. 2010;86(2):192-201. doi:10.1093/cvr/cvp391.

16. Jones BA, Beamer M, Ahmed S. Fractalkine/CX3CL1: a potential new target for inflammatory diseases. Mol Interv. 2010;10(5):263-270. doi:10.1124/ mi.10.53.

17. Juenet M, Varna M, Aid-Launais R, Chauvierre C, Letourneur D. Nanomedicine for the molecular diagnosis of cardiovascular pathologies. Biochem Biophys Res Commun. 2015;468(3):476-484. doi:10.1016/ j.bbrc.2015.06.138.
18. Charoenphol P, Mocherla S, Bouis D, Namdee K, Pinsky DJ, Eniola-Adefeso O. Targeting therapeutics to the vascular wall in atherosclerosis-Carrier size matters. Atherosclerosis. 2011;217(2):364-370. doi:10.1016/j.atherosclerosis. 2011.04.016.

19. Kelly KA, Allport JR, Tsourkas A, Shinde-Patil VR, Josephson L, Weissleder R. Detection of vascular adhesion molecule-1 expression using a novel multimodal nanoparticle. Circ Res. 2005;96(3):327-336. doi:10.1161/ 01.RES.0000155722.17881.dd

20. Edsfeldt A, Grufman H, Asciutto G, et al. Circulating cytokines reflect the expression of pro-inflammatory cytokines in atherosclerotic plaques. Atherosclerosis. 2015;241:443-449. doi:10.1016/j.atherosclerosis.2015.05.019

21. Lobatto ME, Fuster V, Fayad ZA, Mulder WJM. Perspectives and opportunities for nanomedicine in the management of atherosclerosis. Nat Rev Drug Discov. 2011;10(11):835-852. doi:10.1038/nrd3578.

22. Moore KJ, Sheedy FJ, Fisher EA. Macrophages in atherosclerosis: a dynamic balance. Nat Rev Immunol. 2013;13(10):709-721. doi:10.1038/nri3520.

23. Choi HS, Liu W, Misra P, et al. Renal clearance of quantum dots. Nat Biotechnol. 2007:25(10):1165-1170. doi:10.1038/nbt1340.

24. Albanese A, Tang PS, Chan WCW. The Effect of Nanoparticle Size, Shape, and Surface Chemistry on Biological Systems. Annu Rev Biomed Eng. 2012;14(1):1-16. doi:10.1146/annurev-bioeng-071811-150124.

25. Blanco E, Shen H, Ferrari M. Principles of nanoparticle design for overcoming biological barriers to drug delivery. Nat Biotechnol. 2015;33(9):941-951. doi:10.1038/nbt.3330.

26. Hola K, Markova Z, Zoppellaro G, Tucek J, Zboril R. Tailored functionalization of iron oxide nanoparticles for MRI, drug delivery, magnetic separation and immobilization of biosubstances. Biotechnol Adv. 2015;33(6):1162-1176. doi:10.1016/j.biotechadv.2015.02.003.

27. Dung NT, Long NV, Tam LTT, et al. High magnetisation, monodisperse and water-dispersible CoFe@Pt core/shell nanoparticles. Nanoscale. 2017:9(26):8952-8961. doi:10.1039/c6nr09325f.

28. Boyle JJ, Johns M, Kampfer T, et al. Activating transcription factor 1 directs Mhem atheroprotective macrophages through coordinated iron handling and foam cell protection. Circ Res. 2012;110(1):20-33. doi:10.1161/ CIRCRESAHA.111.247577.

29. Boyle JJ, Harrington HA, Piper E, et al. Coronary intraplaque hemorrhage evokes a novel atheroprotective macrophage phenotype. Am J Pathol. 2009;174(3):1097-1108. doi:10.2353/ajpath.2009.080431.

30. Boyle JJ, Christou I, Iqbal MB, et al. Solid-phase immunoglobulins IgG and $\operatorname{IgM}$ activate macrophages with solid-phase IgM acting via a novel scavenger receptor a pathway. Am J Pathol. 2012;181(1):347-361. doi:10.1016/ jajpath.2012.03.040

31. Boyle JJ, Weissberg PL, Bennett MR. Tumor necrosis factor-a promotes macrophage-induced vascular smooth muscle cell apoptosis by direct and autocrine mechanisms. Arterioscler Thromb Vasc Biol. 2003;23(9):1553-1558. doi:10.1161/01.ATV.0000086961.44581.B7.

32. Boyle JJ, Bowyer DE, Weissberg PL, Bennett MR. Human Blood - Derived Macrophages Induce Apoptosis in. Arterioscler Thromb Vasc Biol. 2001:21:1402-1407.

33. Mollet IG, Patel D, Govani FS, et al. Low dose iron treatments induce a DNA damage response in human endothelial cells within minutes. PLoS One. 2016:11(2):1-21. doi:10.1371/journal.pone.0147990.

34. Mohri Z, Rowland EM, Clarke LA, et al. Elevated uptake of plasma macromolecules by regions of arterial wall predisposed to plaque instability in a mouse model. PLoS One. 2014;9(12):1-22. doi:10.1371/journal.pone.0115728.

35. Makowski MR, Varma G, Wiethoff AJ, et al. Noninvasive assessment of atherosclerotic plaque progression in ApoE -/- mice using susceptibility gradient mapping. Circ Cardiovasc Imaging. 2011:4(3):295-303. doi:10.1161/CIRCIMAGING.110.957209.

36. Sun $\mathrm{S}$, Zeng H, Robinson DB, et al. Monodisperse MFe2O4 ( $\mathrm{M}=\mathrm{Fe}, \mathrm{Co}, \mathrm{Mn})$ Nanoparticles. J Am Chem Soc. 2004;126(1):273-279. doi:10.1149/1.3365025.

37. Makowski MR, Wiethoff AJ, Blume U, et al. Assessment of atherosclerotic plaque burden with an elastin-specific magnetic resonance contrast agent. Nat Med. 2011;17(3):383-389. doi:10.1038/nm.2310. 